\title{
三个超高产夏玉米品种的干物质生产及光合特性
}

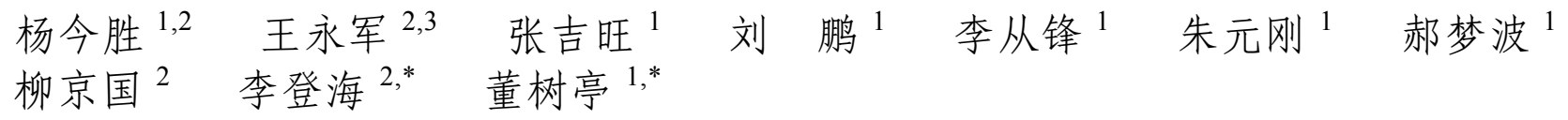

${ }^{1}$ 山东农业大学农学院 / 作物生物学国家重点实验室, 山东泰安 $271018 ;{ }^{2}$ 山东登海种业股份有限公司 / 山东省玉米育种与栽培技术 重点实验室, 山东莱州 $261448 ;^{3}$ 吉林省农业科学院环资中心, 吉林长春 130033

摘 要: 探明不同株高、穗位高具有超高产潜力 $\left(>15000 \mathrm{~kg} \mathrm{hm}^{-2}\right)$ 夏玉米品种的物质生产及光合特性, 有利于密植增 产。本研究在多年高产试验基础上(连续 3 年达 $15000 \mathrm{~kg} \mathrm{hm}^{-2}$ 以上), 选用了登海 661 (DH661, 低秆低穗位)、登海 701 (DH701, 中秆中穗位)和先玉 335 (XY335, 高秆高穗位) 3 个具有超高产潜力的品种进行试验。在一般高产条件下, 这 3 个品种间产量无显著差异, 但干物质生产特性的模型解析表明, DH661 的产量潜力高于 DH701 和 XY335, 其活 跃生长期比 XY335长近 3 周。开花后光合特征参数显示, DH661 光合作用与光能利用效率明显较高, 尤其是当 XY335 进入生理功能速衰期时(开花后 $28 \mathrm{~d}$ ), DH661 仍处于缓慢下降阶段, 且具有较高的光合速率和光能利用效率, DH661 的这一特性表明其具有更高的产量潜力。

关键词：超高产夏玉米; 株高; 穗位高; 物质生产; 光合特性

\section{Dry Matter Production and Photosynthesis Characteristics of Three Hybrids of Maize (Zea mays L.) with Super-High-Yielding Potential}

YANG Jin-Sheng ${ }^{1,2}$, WANG Yong-Jun ${ }^{2,3}$, ZHANG Ji-Wang ${ }^{1}$, LIU Peng ${ }^{1}$, LI Cong-Feng ${ }^{1}$, ZHU Yuan-Gang ${ }^{1}$, HAO Meng-Bo ${ }^{1}$, LIU Jing-Guo ${ }^{2}$, LI Deng-Hai ${ }^{2, *}$, and DONG Shu-Ting ${ }^{1, *}$

\footnotetext{
${ }^{1}$ State Key Laboratory of Crop Biology / College of Agronomy, Shandong Agricultural University, Tai'an 271018, China; ${ }^{2}$ Shandong Denghai Seed-Breeding Co. Ltd. / Key Laboratory of Maize Breeding and Culture of Shandong Province, Laizhou 261448, China; ${ }^{3}$ Research Center of Agricultural Environment and Resources, Jilin Academy of Agriculture Science, Changchun 130033, China
}

\begin{abstract}
The plant height and ear height are the important factors affecting the grain yield in maize (Zea mays L.) which determined the planting density. Obviously, it is essential for high yield to explore the dry matter production and photosynthesis characteristics of super-high-yielding potential hybrid maize with different plant heights and ear heights. Therefore, a field experiment with 82500 plant ha $^{-1}$ was conducted to evaluate the three hybrids (Denghai 661, DH661, the low-plant height and low-ear height; Denghai 701, DH701, the medium-plant height and medium-ear height; Xianyu 335, XY335, the high-plant height and high-ear height). The biomass, leaf area index (LAI), potential photosynthetic capacity (LAD), gas exchange parameters (light-saturated photosynthetic rate- $P_{\text {sat }}$, stomatal conductance- $g_{\mathrm{s}}$ and intercellular $\mathrm{CO}_{2}$ concentration- $C_{\mathrm{i}}$ ) and chlorophyll fluorescence parameters (maximal efficiency of PSII photochemistry- $F_{\mathrm{v}} / F_{\mathrm{m}}$, PSII efficiency- $\Phi_{\mathrm{PSII}}$ and photochemical quenching co-efficient- $q_{\mathrm{P}}$ ) were determined. Yields of the three-type hybrids were over $15000 \mathrm{~kg} \mathrm{ha}^{-1}$, but there were no significant differences among the three hybrids under conventional planting conditions. The results analyzed by Richards model showed that DH661 had the highest yield potential among the three hybrids because its active growth period was about three weeks longer than that of XY335. The yield potential of DH661 was remarkably high from $28 \mathrm{~d}$ after anthesis to harvest stage since it remained the higher photosynthesis capability and radiation utilization efficiency, but that of XY335 decreased rapidly.

Keywords: Super-high-yielding summer maize (Zea mays L.); Plant height; Ear height; Dry matter production; Photosynthesis characteristics
\end{abstract}

密植是挖掘玉米产量潜力最重要和易于调控的 途径之一，而应用株型紧凑、产量潜力大的玉米杂
交种是获得高产的基础 ${ }^{[1-2]}$ 。在玉米上, Mock 提出“理 想株型”育种后, 经历了由“几何株型到生理株型再到

本研究由国家科技支撑计划项目(2007BAD31B04), 山东省泰山学者岗位(ts200648033)和山东省良种产业化项目(2008-7)资助。

“通讯作者(Corresponding authors): 董树亭, E-mail: stdong@sdau.edu.cn, Tel: 0538-8245838; 李登海, Tel: 0535-2788917

第一作者联系方式: E-mail: jsyang1@163.com

Received(收稿日期): 2010-03-25; Accepted(接受日期): 2010-08-06. 
结合了杂种优势的生理生态株型”的演变过程 ${ }^{[-4]}$, 紧 凑株型与杂种优势结合的育种思路在我国的玉米品 种改良中发挥了重要作用。紧凑型玉米增产主要得 益于密植条件下, 较高的群体光合和合理的冠层光 辐射分布与截获 ${ }^{[5-7]}$, 其生态学基础是群体“现实资 源位”向“最适资源位”不断逼近, 使群体利用光资源 进行有效生产能力不断增强的过程 ${ }^{[8-10]}$ 。

在玉米的主要农艺性状中, 株高、穗位高和株 型决定着种植密度, 并在一定程度上影响着玉米的 增产潜力, 因此育种家对株高、穗位高性状的关注 仅次于产量及抗性等指标 ${ }^{[11]}$ 。在玉米品种更替过程 中，产量的提高是生物量和收获指数协同提高的结 果 ${ }^{[12-13]}$, 而收获指数仅从 0.45 提高到 0.50 左右, 这 就意味着进一步提高玉米产量需依赖于光合产物的 更多积累, 以形成更高的生物量 ${ }^{[14]}$ 。显然, 植株越高, 就越利于改善冠层光分布, 适于高产群体构建, 提 高光能截获与利用效率。但在较高密度条件下, 植 株过高无疑会增加倒伏风险, 因此, 为增加种植密 度和提高抗倒伏性, 适当降低株高和穗位高在玉米 高产育种和栽培中值得高度关注。适当降低株高使 光截获中心下移对提高群体适应性, 增强抗逆能力 具有积极意义 ${ }^{[15-16]}$ 。株高降低, 将使冠层纵向空间 生态位变窄, 这就要求在有限的生长季节增加种植密 度时确保群体最大叶面积不超过临界值, 必须同时提 高源器官光合效能和高值持续时间, 开拓潜在时间生 态位以提高群体光能利用率, 增加光合物质积累 ${ }^{[17]}$ 。

株高、穗位高对玉米产量具有显著正效应 ${ }^{[18-20]}$, 但又与高产存在矛盾。对不同株高类型的品种在不 同密度下的研究认为, 中等株高的品种对密度反映 适中, 理论产量高 ${ }^{[21]}$ 。然而, 在高密度条件下, 具有 超高产潜力的品种是否也表现同样规律 ? 尽管黄振 喜等 ${ }^{[22]}$ 选用 3 个具有 $15000 \mathrm{~kg} \mathrm{hm}^{-2}$ 高产潜力的杂交 种在 78000 株 $\mathrm{hm}^{-2}$ 密度下种植, 认为籽粒灌浆启动 快且高灌浆速率持续时间和生长活跃期长的杂交种 更容易实现高产, 并阐释了其碳代谢的酶学机制, 但尚缺乏针对不同株高、穗位高具有超高产潜力品 种的相关研究。本研究将在高密度条件下, 进一步 探讨株高、穗位高不同的 3 个超高产夏玉米品种的 物质生产和光合特性, 旨在丰富超高产玉米育种理 论, 为超高产栽培提供科学依据。

\section{1 材料与方法}

\section{1 试验材料与试验设计}

在国家玉米工程技术研究中心(山东)的高产试
验田(东经 $119^{\circ} 56.6^{\prime}$, 北纬 $37^{\circ} 20.7^{\prime}$, 海拔 $8.1 \mathrm{~m}$ )进行 试验。选用 3 个不同株高、穗位高, 具有超高产潜力 的玉米杂交种先玉 335 (XY335)、登海 661 (DH661) 和登海 701 (DH701), 上述 3 品种在 2006-2008 连 续 3 年高产攻关中实现了 $15000 \mathrm{~kg} \mathrm{hm}^{-2}$ 的产量目标 (表 1)。多年高产攻关篮选表明, 这 3 个杂交种在高 产攻关栽培条件下生育期基本一致。2006 年和 2007 年均为 6 月 15 日播种, 10 月 15 日收获; 2008 年于 6 月 14 日播种, 10 月 7 日收获, 受气候影响收获时已 达生理成熟。种植密度均为 82500 株 $\mathrm{hm}^{-2}$, 株距 20.2 $\mathrm{cm}$, 行距 $60 \mathrm{~cm}$, 小区面积 $80 \mathrm{~m}^{2}$, 随机区组设计, 3 次重复。试验地前茬作物为冬小麦, 沙壤土, 土壤 0 20 cm 耕层含有机质 $1.15 \%$ 、全氮 $0.096 \%$ 、有效 氮 $69.7 \mathrm{mg} \mathrm{kg}^{-1}$ 、速效磷 $48.5 \mathrm{mg} \mathrm{kg}^{-1}$ 、速效钾 118.6 $\mathrm{mg} \mathrm{kg}{ }^{-1}$ 。按一般高产攻关田管理, 生育期内施用 $\mathrm{N}$ $450 \mathrm{~kg} \mathrm{hm}^{-2}, \mathrm{P}_{2} \mathrm{O}_{5} 225 \mathrm{~kg} \mathrm{hm}^{-2}, \mathrm{~K}_{2} \mathrm{O} 300 \mathrm{~kg} \mathrm{hm}^{-2}$, 其 中氮肥按 $2: 1: 2$ 比例分别做底肥、大喇叭口期和 授粉后追肥，磷钾肥做底肥一次施入。

1.2 测定内容及方法

1.2.1 䊏粒产量及收获指数 收获期, 在每小区 连续收取中间 3 行, 收获 $18 \mathrm{~m}^{2}$ 面积上的果穗, 脱粒, 烘干, 计产; 在每小区连续取样 10 株, 烘干至恒重, 计算收获指数。

\subsection{2 千物质积累过程拟合 以播种后天数 $(t)$ 为} 自变量, 以播种后 $0 、 28 、 59 、 72 、 93$ 和 $115 \mathrm{~d}$ 测得 单株地上部干重为因变量 $(W)$, 用 Richards 方程 $W=$ $A\left(1+B \mathrm{e}^{-C t}\right)^{-1 / D}$ (当 $D=1$ 时, 为 Logistic 方程)对干物质 积累过程进行模拟 ${ }^{[23-24]}$ 。干物质积累速率最大时的 日期 $T_{\text {max }}=(\ln B-\ln D) / c$, 干物质积累速率最大时的 生长量 $W_{\text {max }}=A(D+1)^{-1 / D}$, 最大干物质积累速率 $G_{\max }$ $=\left(C W_{\max } / D\right)\left[1-\left(W_{\max } / A\right)^{D}\right]$, 生长活跃期(大约完成总 积累量的 $90 \%) P=2(D+2) / C$ 。对生长曲线求导, 得干 物质积累速率 $\left(\mathrm{g}_{\text {株 }}^{-1} \mathrm{~d}^{-1}\right)$ 。

1.2.3 叶面积及光合势 播种后 $0 、 28 、 59 、 72$ 、 93 和 $115 \mathrm{~d}$ 测定叶面积, $L A=l$ (叶片最大长度) $\times w$ (最 大宽度 $) \times 0.75$, 计算叶面积指数 $(\mathrm{LAI})$; 参照赵会杰 等 ${ }^{[25]}$ 的方法计算光合势 $L A D=(L A 1+L A 2) / 2 \times(t 2-t 1)$, 单位为 $\mathrm{m}^{2} \mathrm{hm}^{-2} \mathrm{~d}^{-1}$ 。

1.2.4 叶片光合速率及气体交换参数 于开花授 粉前选择生长一致的代表性植株挂牌标记，自开花 之日起，于花后 $0 、 18 、 28 、 58 \mathrm{~d}$ 用 Ciras-II 便携式 光合测定系统(PPSystem, UK) 测定果穗叶饱和光合 速率 $\left(P_{\text {sat }}\right)$ 。选择晴朗无云天气的上午 10:00 12:00 
测定, 采用开放式气路, 冠层果穗叶附近 $\mathrm{CO}_{2}$ 浓度 $360 \sim 380 \mu \mathrm{mol} \mathrm{mol}^{-1}$, 采用系统 LED 光源补光, 光量子 通量为 $1600 \mu \mathrm{mol} \mathrm{m} \mathrm{m}^{-2} \mathrm{~s}^{-1}$ 。气孔导度 $\left(G_{\mathrm{s}}\right)$ 和细胞间隙 $\mathrm{CO}_{2}$ 浓度 $\left(C_{\mathrm{i}}\right)$ 由系统测得的 $P_{\text {sat }}$ 、叶温等参数计算得出。 1.2.5 叶片叶绿素荧光参数在测定光合的同时, 采用 FMS2 脉冲调制式苂光仪(Hansatech, UK) 测定 相应果穗叶的叶绿素荣光各参数。各参数的意义及 计算公式如下 ${ }^{[26-27]}$, PSII 的最大光化学效率为 $F_{\mathrm{v}} / F_{\mathrm{m}}$; 实际光化学效率 $\Phi_{\mathrm{PSII}}=q_{\mathrm{P}} \times\left(F_{\mathrm{v}}{ }^{\prime} / F_{\mathrm{m}}{ }^{\prime}\right)=\left(F_{\mathrm{m}}{ }^{\prime}-F_{\mathrm{s}}\right) / F_{\mathrm{m}}{ }^{\prime}$; 光 化学猝灭 $q_{\mathrm{P}}=\left(F_{\mathrm{m}}{ }^{\prime}-F_{\mathrm{s}}\right) /\left(F_{\mathrm{m}}{ }^{\prime}-F_{\mathrm{o}}{ }^{\prime}\right)$ 。其中, $F_{\mathrm{o}}{ }^{\prime}$ 为光适应 后的初始荧光, $F_{\mathrm{m}}{ }^{\prime}$ 为光适应后的最大荧光, $F_{\mathrm{o}}$ 为暗适 应后的初始荧光, $F_{\mathrm{m}}$ 为暗适应后的最大荧光, $F_{\mathrm{s}}$ 为光 适应后的稳态苂光, $F_{\mathrm{v}}{ }^{\prime}$ 为光适应后的最大可变苂光。

\section{3 数据处理与统计分析}

用 Microsoft Excel 2003 进行数据计算; 用 SPSS 11.0 统计软件进行方差分析; 用 SigmaPlot 10.0 作图, 并进行生长曲线模拟与解析。

\section{2 结果与分析}

\section{1 不同类型玉米品种的产量与植株性状}

3 个株高、穗位高不同的超高产玉米品种籽粒产 量连续 3 年达 $15000 \mathrm{~kg} \mathrm{hm}^{-2}$ 以上产量目标, 但 3 个 品种间籽粒产量与收获指数均差异不显著 $(P<0.05)$ (表 1)。其中, 以登海 661 (DH661)株高、穗位高均 最低, 而株高/穗位高值最高(3.10 以上), 其穗上节
间相对拉长程度最高。

\section{2 干物质积累特性}

由图 1 可看出, 3 个品种地上部干物质积累过程 存在明显的两段快速增长期，一是拔节期至开花期 (播种后 28 59 d), 二是灌浆盛期至收获期(播种后 $72 \sim 115 \mathrm{~d})$ 。整个干物质积累过程中, 品种间并未表 现出明显差异, 但在干物质积累速率上, XY335 表 现出早发趋势, 而 DH661 和 DH701 则在籽粒产量 形成的后期下降相对较慢。3 个品种的物质积累过程 可用 Logistic 生长方程较好拟合 $\left(R^{2}=0.976 \sim 0.988\right)$, XY335 达到最大生长速率时所用天数明显低于 DH661 和 DH701, 分别减少 $21.36 \mathrm{~d}$ 和 $12.15 \mathrm{~d}$, 但其 生长速率较高, 早发优势明显。终极生长量以 DH661 和 DH701 高于 XY335, 其生长活跃期明显较 长, 分别比 XY335 多 $19.67 \mathrm{~d}$ 和 $13.24 \mathrm{~d}$, 按模型得 到的生长活跃天数推算, DH661、DH701 和 XY335 的物质生产潜力比收获时实际干重分别高 $18.3 \%$ 、 $10.4 \%$ 和 3.5\%，表明 DH661 的产量潜力较大(表 2)。

\section{3 叶面积指数变化及光合势}

在群体叶面积建成与衰退过程中, DH661 的光 合面积始终相对较高, 播种后 $90 \mathrm{~d}, \mathrm{XY} 335$ 的 LAI 表现快速下降趋势, 而 DH661 和 DH701 则下降缓 慢。3 个品种开花后光合势占总光合势的 $57 \%$ 60\%, 其中 DH661 的总光合潜力较大，在籽粒产量形成期 间尤为明显(图 2)。

表 1 供试品种的产量及植株性状

Table 1 Yield and plant traits of the hybrid maize in the study

\begin{tabular}{|c|c|c|c|c|c|c|}
\hline $\begin{array}{l}\text { 年份 } \\
\text { Year }\end{array}$ & $\begin{array}{l}\text { 品种 } \\
\text { Hybrid }\end{array}$ & $\begin{array}{c}\text { 产量 } \\
\text { Yield } \\
\left(\mathrm{kg} \mathrm{hm}^{-2}\right) \\
\end{array}$ & $\begin{array}{c}\text { 收获指数 } \\
\text { Harvest index }\end{array}$ & $\begin{array}{c}\text { 株高 } \\
\text { Plant height } \\
(\mathrm{cm})\end{array}$ & $\begin{array}{c}\text { 穗位高 } \\
\text { Ear height } \\
(\mathrm{cm})\end{array}$ & $\begin{array}{c}\text { 株高/穗位高 } \\
\text { Plant height/ } \\
\text { ear height }\end{array}$ \\
\hline \multirow[t]{3}{*}{2006} & 登海 661 DH661 & 17261 & 0.54 & 242 & 78 & $3.10: 1$ \\
\hline & 登海 701 DH701 & - & 0.54 & 269 & 92 & $2.92: 1$ \\
\hline & 先玉 335 XY335 & 17056 & 0.53 & 286 & 103 & $2.78: 1$ \\
\hline \multirow[t]{3}{*}{2007} & 登海 661 DH661 & 17561 & 0.54 & 242 & 76 & $3.18: 1$ \\
\hline & 登海 701 DH701 & 17533 & 0.55 & 280 & 93 & $3.01: 1$ \\
\hline & 先玉 335 XY335 & 17015 & 0.53 & 288 & 104 & $2.77: 1$ \\
\hline \multirow[t]{3}{*}{2008} & 登海 661 DH661 & $16202 \mathrm{a}$ & $0.55 \mathrm{a}$ & $241 \mathrm{c}$ & $76 \mathrm{c}$ & $3.17: 1$ \\
\hline & 登海 701 DH701 & $16905 \mathrm{a}$ & $0.56 \mathrm{a}$ & $277 \mathrm{~b}$ & $93 \mathrm{~b}$ & $2.98: 1$ \\
\hline & 先玉 335 XY335 & $16423 \mathrm{a}$ & $0.53 \mathrm{a}$ & $288 \mathrm{a}$ & $104 \mathrm{a}$ & $2.77: 1$ \\
\hline
\end{tabular}

DH661 在 2006 年和 2007 年的产量为实打验收, 其他均为小区试验结果, 种子含 $14 \%$ 的标准水分; 标以相同小写字母的值表示 在 0.05 水平, 差异不显著。

The yield of DH661 was the actual yield in 2006 and 2007; the others were estimated from the plot yield. The yield was estimated as that contained $14 \%$ moisture. Values followed by the same letters are not significantly different at the 0.05 probability level. 


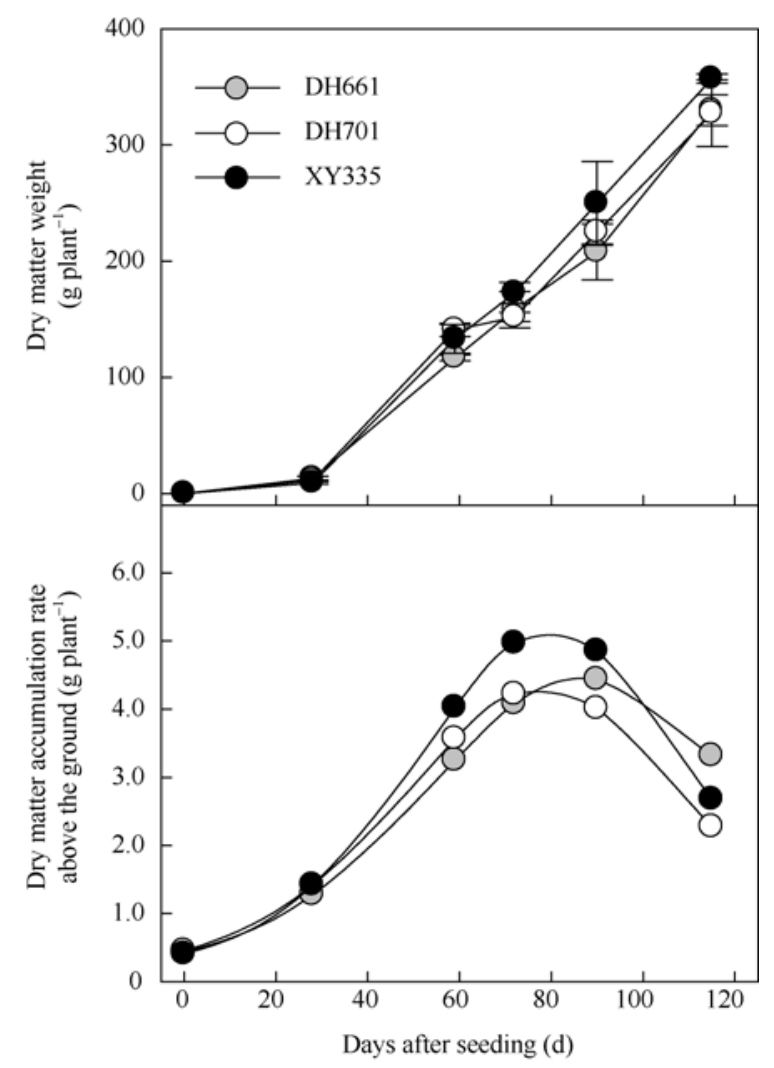

图 1 不同品种超高产玉米干物质积累动态及积累速率

Fig. 1 The grain yield and harvest index among different hybrids with super-high-yielding potential

\section{4 叶片光合速率及相关参数}

图 3 表明, 从开花期到开花后 $28 \mathrm{~d}, 3$ 个超高产 玉米品种的饱和光合速率、气孔导度均差异不大, 而从开花后 $28 \mathrm{~d}$ 到收获期, XY335 的下降最快, DH661 最慢。细胞间隙 $\mathrm{CO}_{2}$ 浓度的变化则表现相反 的趋势, 而且籽粒产量形成前期即表现出较高趋势, 反映出叶肉细胞 $\mathrm{CO}_{2}$ 同化能力的差异, XY335 相对 较低, 且越到籽粒产量形成后期越为明显。

2.5 叶片叶绿素荧光特性

由图 4 可见, 3 个超高产玉米品种的最大光化学
效率、实际光化学效率和光化学猝灭表现出相同的 变化趋势, 即从开花期到开花后 $28 \mathrm{~d}$ 期间差异不大, 而从开花后 $28 \mathrm{~d}$ 到收获期, XY335 的下降较快, DH661 下降较慢。表明 3 个超高产玉米品种在叶片 衰老过程中, PSII 捕获光能的利用效率下降具明显 差异，这导致光合速率降低的进度有所不同。

\section{3 讨论}

在玉米的农艺性状中, 株高、穗位高和果穗性 状与产量关系尤为密切 ${ }^{[11,18-20]}$, 当代高产品种植株 和穗位变高, 茎秆变粗, 耐密性增强, 抗病、抗倒伏 能力增强从而使其更具高产稳产特性 ${ }^{[28]}$ 。另有研究 认为, 现代玉米品种较高的耐密能力主要是因为其 冠层结构紧凑, 植株矮化, 叶片少而直, 使冠层在 较高的叶面积指数下, 仍能截获到较多的光照, 而 且通过降低结穗高度, 提高了茎秆抗倒伏能力, 这 些特征都有助于现代品种在高密度下获得较高的产 量 ${ }^{[29]}$ 。为阐释当前玉米品种植株相对高大还是植株 相对矮小更有利于高产, 本研究选用 3 个株高、穗位 高不同的超高产玉米品种在常规高产攻关田种植, 均达到 $15000 \mathrm{~kg} \mathrm{hm}^{-2}$ 的产量, 但三品种间差异不显 著。然而, 这是否表明其产量与株高、穗位高无关 呢? 以 Richards 方程进一步解析后发现, 登海 661 (DH661), 在生育后期物质生产潜力较大, 其生长活 跃期显著高于登海 701 (DH701)和先玉 335 (XY335), 按模型得到的生长活跃天数推算, DH661、DH701 和 XY335 的干物质生产潜力比收获时实际干物质积 累量分别高 $18.3 \%$ 、10.4\%和 $3.5 \%$ (表 2)。

前人研究认为, 玉米对密度耐受能力的提高是 在高密度条件下大范围高产育种的附带效应 ${ }^{[29-30]}$, 而不是对种植密度适应力直接选择的结果 ${ }^{[31]}$ 。对株 高、穗位高的直接选择, 主要目标是解决玉米生产 中的倒伏问题。前人针对玉米在干旱等逆境条件下

表 2 不同品种超高产玉米干物质生产特征参数

Table 2 Characteristic parameters of dry matter accumulation in different hybrids with super high-yielding potential

\begin{tabular}{cccccccccc}
\hline $\begin{array}{c}\text { 品种 } \\
\text { Hybrid }\end{array}$ & $R^{2}$ & $\mathrm{~A}$ & $\mathrm{~B}$ & $\mathrm{C}$ & $\begin{array}{c}\mathrm{T}_{\max } \\
(\mathrm{d})\end{array}$ & $\begin{array}{c}\mathrm{W}_{\max } \\
\left(\mathrm{g} \mathrm{plant}^{-1}\right)\end{array}$ & $\begin{array}{c}\mathrm{G}_{\max } \\
\left(\mathrm{g} \mathrm{plant}^{-1} \mathrm{~d}^{-1}\right)\end{array}$ & $\begin{array}{c}\mathrm{P} \\
(\mathrm{d})\end{array}$ & $\begin{array}{c}\mathrm{DW}_{\mathrm{P}} \\
\left(\mathrm{g} \mathrm{plant} \mathrm{t}^{-1}\right)\end{array}$ \\
\hline DH661 & 0.984 & 419.45 & 39.12 & 0.043 & 141.98 & 209.73 & 4.46 & 141.06 & 383.67 \\
DH701 & 0.976 & 417.18 & 36.07 & 0.045 & 132.77 & 194.09 & 4.36 & 133.63 & 355.17 \\
XY335 & 0.988 & 408.06 & 49.28 & 0.049 & 120.62 & 204.03 & 5.08 & 120.39 & 364.08 \\
\hline
\end{tabular}

$R^{2}$ 为决定系数; $\mathrm{A}$ : 终极生长量; $\mathrm{B}$ : 初值参数; $\mathrm{C}$ : 生长速率参数。 $\mathrm{DW}_{\mathrm{P}}$ : 生育天数达到活跃生长期 $\mathrm{P}$ 值时推算的单株干物质积累 量潜力。

$R^{2}$ : the coefficient of determination; A: the maximum growth biomass; B: the initial parameter; C: the growth rate parameter. $\mathrm{DW}_{\mathrm{P}}$ : the dry matter accumulation potential calculated by Richards model at the days of development to the active growing stage (P). 

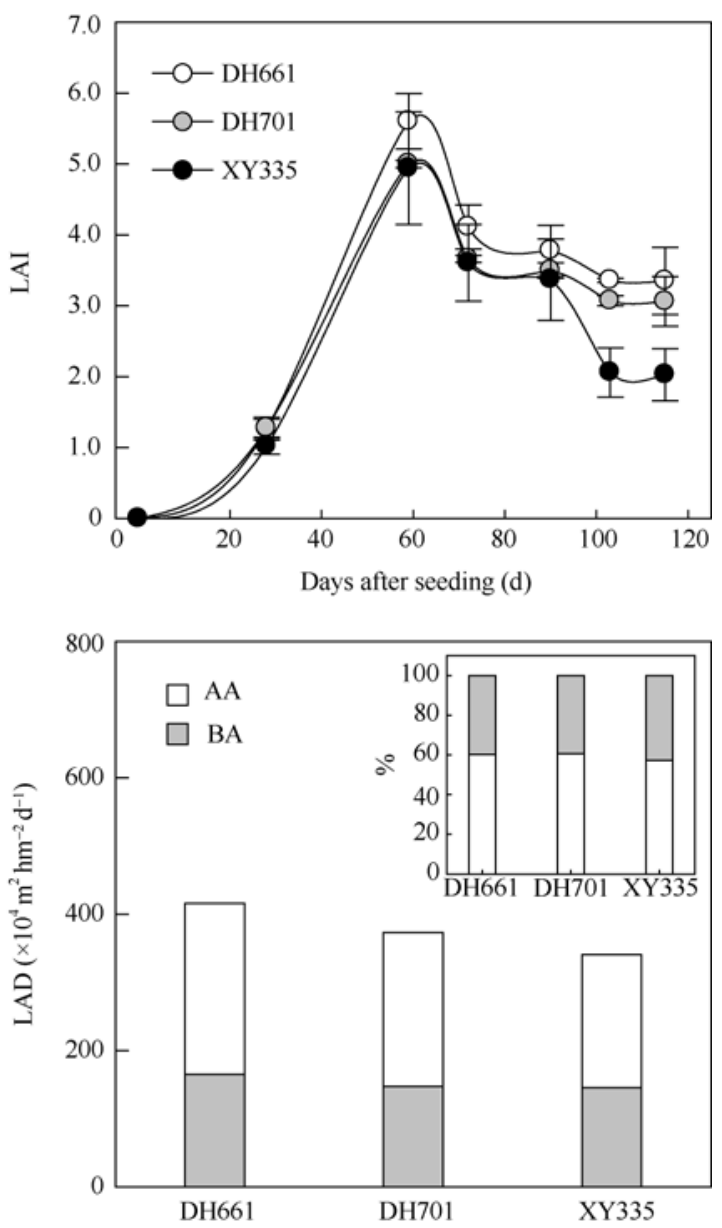

图 2 不同品种超高产玉米叶面积指数(LAI)动态及光合势 (LAD) 比较

Fig. 2 Dynamics of leaf area index (LAI) and the comparison of potential photosynthetic capacity (LAD) among different hybrids with super-high-yielding potential $\mathrm{AA}$ ：开花后; BA: 开花前。

AA: the phase after anthesis; BA: the phase before anthesis.

的研究认为, 适当降低株高, 可提高群体适应性, 增强抗逆能力 ${ }^{[15-16]}$, 而在本研究较为优越的栽培条 件下, 尽管 3 个超高产品种籽粒产量无显著差异, 但 DH661 表现出较大的增产潜力(表 2、图 2、图 3 和 图 4)。然而, 高产品种的株高、穗位高多大才有利于 高产形成呢？早在 20 世纪 60 年代, Duncan 等 ${ }^{[32-33]}$ 就 在不同密度和产量条件下研究过高产群体的合理株 高, 他们认为在互相遮阴的群体内, 必然存在一个 为获得最大光截获的最大株高, 但受当时品种特性 和产量水平所限, 不宜作为我国当前高产品种选育 与高产栽培实践的参考。在本研究中, 当品种株高、 穗位高均适当降低后(DH661), 宜适当提高株高/穗 位高比值(3.10 3.18), 显然, 这种穗上节间相对拉 长的株型结构可改善群体冠层光分布, 保持“棒三 叶”较好的受光姿态, 利于高产。

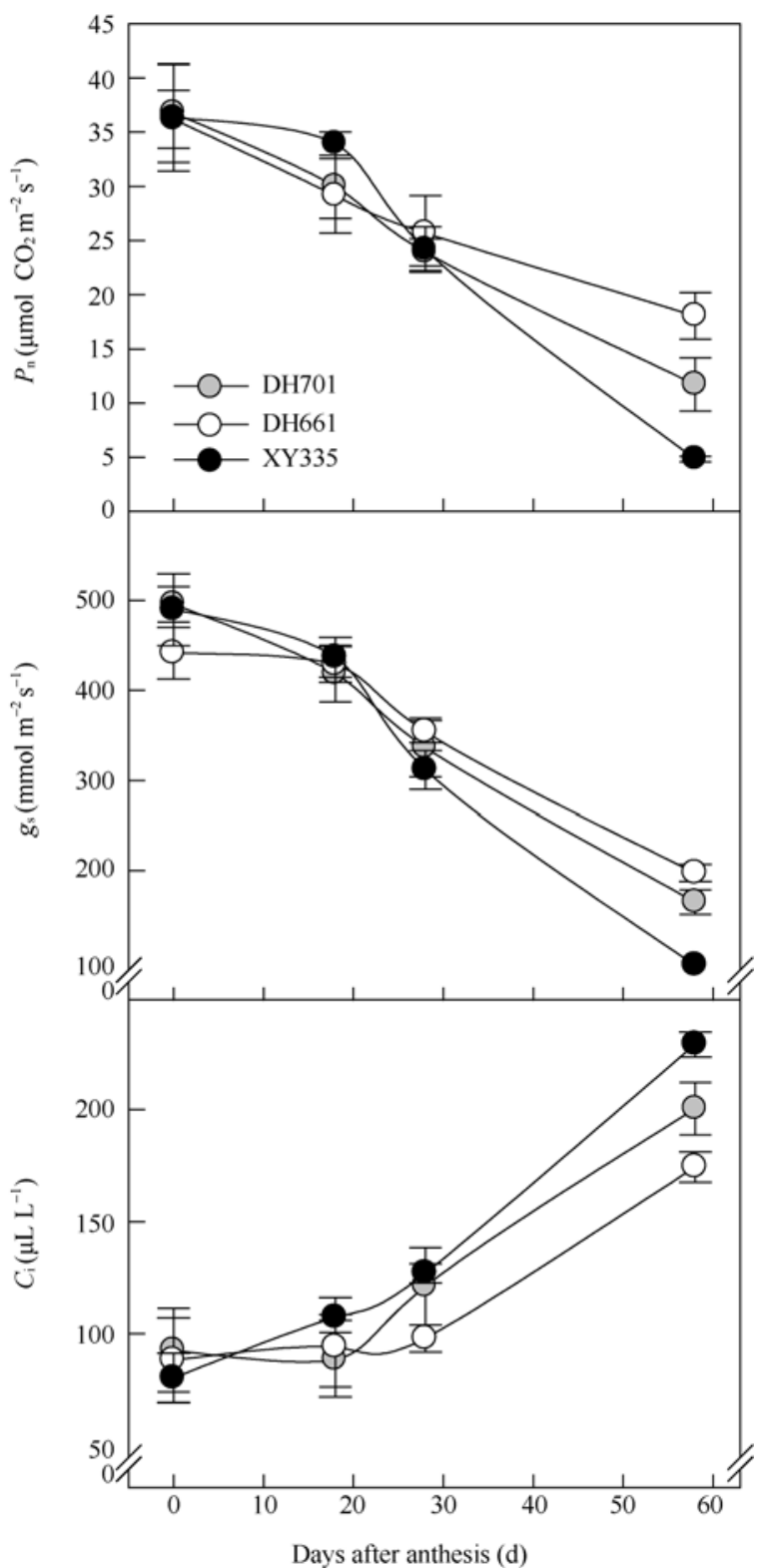

图 3 开花后不同品种超高产玉米果穗叶饱和光合速率 $\left(\boldsymbol{P}_{\mathrm{sat}}\right)$ 、气 孔导度 $\left(G_{\mathrm{s}}\right)$ 和细胞间隙 $\mathrm{CO}_{2}\left(C_{\mathrm{i}}\right)$ 浓度动态

Fig. 3 Dynamics of light-saturated photosynthetic rate $\left(P_{\text {sat }}\right)$, stomatal conductance $\left(G_{s}\right)$, and intercellular $\mathrm{CO}_{2}$ concentration $\left(C_{\mathrm{i}}\right)$ of the ear leaf among different hybrids with super-high-yielding potential after anthesis

作物高产挖潜途径研究 ${ }^{[34]}$ 指出, 夏玉米超高产 以“群体结构性获得”为主要突破途径, 而在高密度 群体中进一步挖掘“个体功能性获得”将是玉米超高 产育种和栽培的主要目标。作物的产量基本取决于 光合机构的大小和效率 ${ }^{[12-13]}$, 所以增强个体功能主 要是提高源器官的光合效率。本研究对光合特性的 比较, 有助于对干物质积累关键过程的理解, 利于 进一步挖掘产量潜力。开花后尤其是开花后 $28 \mathrm{~d}$, DH661 的 LAI 下降最为缓慢, 这势必影响其籽粒产 


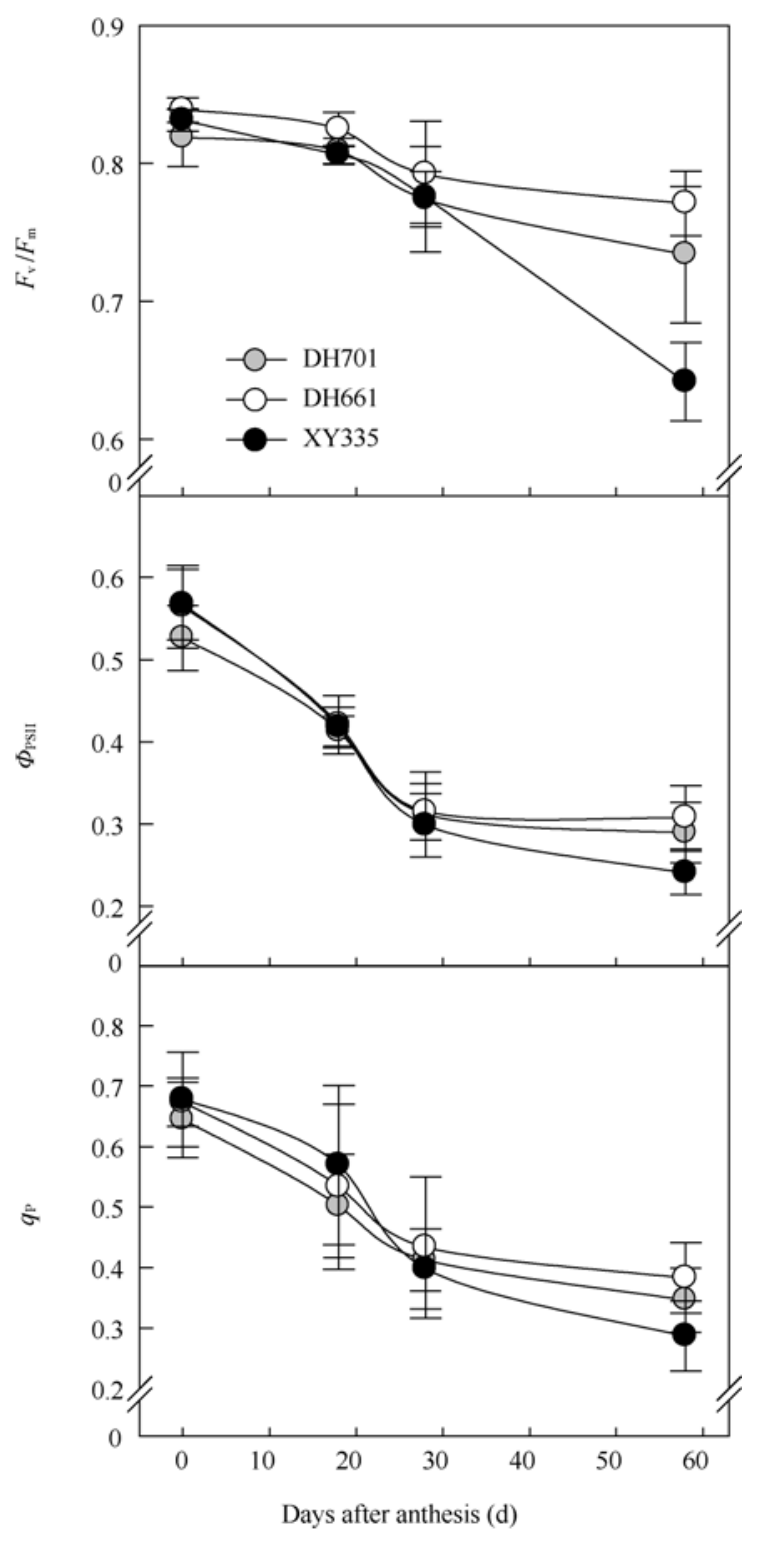

图 4 开花后不同品种超高产玉米果穗叶最大光化学效率 $\left(F_{\mathrm{v}} / F_{\mathrm{m}}\right)$ 、实际光化学效率 $\left(\Phi_{\mathrm{PSII}}\right)$ 和光化学猝灭 $\left(q_{\mathrm{P}}\right)$ 的动态

Fig. 4 Dynamics of maximal efficiency of PSII photochemistry $\left(F_{\mathrm{v}} / F_{\mathrm{m}}\right)$, PSII efficiency ( $\left.\Phi_{\mathrm{PSII}}\right)$, and photochemical quenching co-efficient $\left(q_{\mathrm{P}}\right)$ of the ear leaf among different hybrids with super-high-yielding potential after anthesis

量的形成。DH661 光合碳同化能力和光能利用效率 潜力显著高于其他 2 个品种, 这一优势的突显在开花 后 $28 \mathrm{~d}$ (4 周)后尤其明显。前人研究表明, 在逆境条 件下，吐丝后 2 3 周是玉米对环境压力的高度敏感 期, 该阶段的生理功能决定着最终产量 ${ }^{[35]}$, 本研究 表明, 开花后 4 周到收获期间的光合性能对籽粒产 量形成亦至关重要。所以, 玉米超高产育种要重视 营养生长阶段形态结构快速建成与生殖生长阶段光 合器官衰老缓慢材料的选用; 超高产栽培中应注重 生育后期高产群体光合器官生产功能的维持, 以充
分发挥品种增产潜力。

\section{4 结论}

登海 661 (DH661)具有更高的产量潜力, 其活 跃生长期比先玉 335 (XY335)长近 3 周, 其增产优势 在籽粒形成后期尤为明显。DH661 高产潜力大的生 理基础是当先玉 335 进入生理功能速衰期(开花后 28 d)时, DH661 仍处于缓慢下降阶段, 且具有较高的光 合速率和光能利用效率。

\section{References}

[1] Li D-H(李登海). A review and prospect of erectophile maize breeding. J Laiyang Agric Coll (莱阳农学院学报), 2001, 18(1): 1-6 (in Chinese)

[2] Li D-H(李登海), Mao L-H(毛丽华), Jiang W-J(姜伟娟), Liu $\mathrm{J}-\mathrm{G}$ (柳京国), Li C-M(李春明). Researches on high-yielding performances of erectophile maize hybrids. J Laiyang Agric Coll (莱 阳农学院学报), 2001, 18(4): 259-262 (in Chinese)

[3] Mock J J, Pearce R B. An ideotype of maize. Euphytica, 1975, 24: 613-623

[4] Yu H-F(于洪飞), Dai J-Y(戴俊英), Shen X-Y(沈秀瑛), Zhang $\mathrm{L}$ (张烈). Progress of maize ideotype breeding based on eco-physiology. J Maize Sci (玉米科学), 1995, 3(1): 12-17 (in Chinese)

[5] Winter S R, Ohlrogg A J. Leaf angle, leaf area, and corn (Zea mays L.) yield. Agron J, 1973, 65: 395-397

[6] Xu Q-Z(徐庆章), Wang Q-C(王庆成), Niu Y-Z(牛玉贞), Wang Z-X(王忠孝), Zhang J(张军). Studies on relation between plant type and canopy photosynthesis in maize. Acta Agron Sin (作物 学报), 1995, 21(4): 492-496 (in Chinese with English abstract)

[7] Wang Q-C(王庆成), Niu Y-Z(牛玉贞), Xu Q-Z(徐庆章), Wang Z-X(王忠孝), Zhang J(张军). Effect of plant-type on rate of canopy apparent photosynthesis and yield in maize (Zea mays L.). Acta Agron Sin (作物学报), 1996, 22(2): 223-227 (in Chinese with English abstract)

[8] Li Z-Z(李自珍), Zhao S-L(赵松岭), Zhang P-Y(张鹏云). The niche-fitness theory and its application to the systems of crop growth. J Lanzhou Univ (Nat Sci Edn) (兰州大学学报·自然科学 版), 1993, 29(4): 219-224 (in Chinese with English abstract)

[9] Li Z-Z(李自珍), Li W-L(李文龙). The effect of water-fertilizer conditions on crop's niche-fitness and yield in semi-arid regions of the Loess Plateau. Acta Bot Boreali-Occident Sin (西北植物学 报), 2003, 23(1): 28-33 (in Chinese with English abstract)

[10] Li J(李娎), Zhu J-Z(朱金兆), Zhu Q-K(朱清科). A review on niche theory and niche metrics. J Beijing For Univ (北京林业大 学学报), 2003, 25(1): 100-107 (in Chinese with English abstract)

[11] Duvick D N. Heterosis: feeding people and protecting natural resources. In: Coors J G, Pandey S, eds. Genetics and Exploitation of Heterosis in Crops. Wisconsin: Am Soc Agron, Crop Sci Soc 
Am, Soil Sci Soc Am, Inc, 1999. pp 19-29

[12] Gardner F P, Pearce R B, Mitchell R L. Photosynthesis. In: Physiology of Crop Plant. Ames: Iowa State University Press, 1985. pp 3-30

[13] Fageria N K, Baligar V C, Clark R B. Physiology of Crop Production. Food Products Press. An Imprint of the Haworth Press, Inc. New York, London, Oxford, 2005. pp 72-82

[14] Russell W A. Evaluations for plant, ear, and grain traits of maize cultivars representing seven years of breeding. Maydica, 1985, 30: 85-96

[15] Fischer K S, Johnson E C, Edmeades G O. Breeding and Selection for Drought Resistance in Tropical Maize. CIMMYT, El Batan, Mexico, 1983. pp 396-399

[16] Chapman S C, Edmeades G O. Selection improves drought tolerance in tropical maize populations: II. Direct and correlated responses among secondary traits. Crop Sci, 1999, 39: 1315-1324

[17] Ling Q-H(凌启鸿). Crops Population Quality (作物群体质量). Shanghai: Shanghai Scientific and Technical Publishers, 2000. pp 7-12 (in Chinese)

[18] Tan H-P(谭禾平), Wang G-Y(王桂跃), Hu X-N(胡贤女), Xu $\mathrm{Q}-\mathrm{X}$ (许巧贤). Multiple regression and path analysis of effective factors affecting maize yield. Acta Agric Zhejiangensis (浙江农 业学报), 2006, 18(4): 238-240 (in Chinese with English abstract)

[19] Pereira M G, Lee M. Identification of genomic regions affecting plant height in sorghum and maize. Theor Appl Genet, 1995, 90: 380-388

[20] Zhang Z-M(张泽民), Jia C-Z(贾长柱). Effects of plant-type on genetic gain in maize. Heredita (遗传), 1997, 19(2): 31-34 (in Chinese with English abstract)

[21] Yang L-H(杨利华), Zhang L-H(张丽华), Yang S-L(杨世丽), Ma R-K(马瑞昆), Zhang Q-G(张全国). Responses of some population quality indices of corn hybrids differing in plant height to planting density. Acta Agric Boreal-Sin (华北农学报), 2007, 22(6): 139-146 (in Chinese with English abstract)

[22] Huang Z-X(黄振喜), Wang Y-J(王永军), Wang K-J(王空军), Li D-H(李登海), Zhao M(赵明), Liu J-G(柳京国), Dong S-T(董树 亭), Wang H-J(王洪军), Wang J-H(王军海), Yang J-S(杨今胜). Photosynthetic traits of over- $15000 \mathrm{~kg} \mathrm{ha}^{-1}$ summer maize hybrids during grain filling. Sci Agric Sin (中国农业科学), 2007, 40(9): 1898-1906 (in Chinese with English abstract)

[23] Zhu Q-S(朱庆森), Cao X-Z(曹显祖), Luo Y-Q(骆亦其). Growth analysis on the process of grain filling in rice. Acta Agron Sin (作 物学报), 1988, 14(3): 182-193 (in Chinese with English abstract)

[24] Gu S-L(顾世梁), Zhu Q-S(朱庆森), Yang J-C(杨建昌), Peng
$\mathrm{S}-\mathrm{B}$ (彭少兵). Analysis on grain filling characteristics for different rice types. Acta Agron Sin (作物学报), 2001, 27(1): 7-14 (in Chinese with English abstract)

[25] Zhao H-J(赵会杰), Li Y(李有), Zou Q(邹琦). A comparative study on characteristics of radiation and photosynthesis in canopy of two different spike-type cultivars of wheat. Acta Agron Sin (作 物学报), 2002, 28(5): 654-659 (in Chinese with English abstract)

[26] Demmig-Adams B, Adams W W III. Using chlorophyll fluorescence to assess the fraction of absorbed light allocated to thermal dissipation of excess excitation. Physiol Plant, 1996, 98: 253-264

[27] Genty B, Briantais J M, Baker N R. The relationship between the quantum yield of photosynthetic electron transport and quenching of chlorophyll fluorescence. Biochim Biophys Acta, 1989, 990: $87-92$

[28] Hu C-H(胡昌浩), Dong S-T(董树亭), Wang K-J(王空军), Sun Q-Q(孙庆泉). Studies on development rule for growth characteristics of maize varieties from different eras in China: I. The development of grain yield characteristics. J Maize Sci (玉米科学), 1998, 6(2): 44-48 (in Chinese)

[29] Sangoi L, Gracietti M A, Rampazzo C, Bianchetti P. Response of Brazilian maize hybrids from different areas to changes in plant density. Field Crops Res, 2002, 79: 39-51

[30] Sangoi L, Salvador R J. Effect of maize plant detasseling on grain yield, tolerance to high plant density and drought stress. Pesquisa Agropecuária Brasileira, 1998, 33: 677-684

[31] Tollenaar M, Wu J. Yield improvement in temperate maize is attributable to greater stress tolerance. Crop Sci, 1999, 39: 1579-1604

[32] Duncan W G. Cultural manipulation for higher yields. In: Eastin J D, Haskins F A, Sullivan C Y, Van Bavel C H M, Dinauer R C, eds. Physiological Aspects of Crop Yield. Madison, Wisconsin: American Society of Agronomy \& Crop Science Society of America, 1969. pp 327-342

[33] Williams W A, Loomis R S, Duncan W G, Dovrat A, Nunez F. Canopy architecture at various population densities and the growth and grain yield of corn. Crop Sci, 1968, 8: 303-308

[34] Zhao M(赵明), Li J-G(李建国), Zhang B(张宾), Dong Z-Q(董志 强), Wang M-Y(王美云). The compensatory mechanism in exploring crop production potential. Acta Agron Sin (作物学报), 2006, 32(10): 1566-1573 (in Chinese with English abstract)

[35] Paponov I A, Sambo P, Erley G S A, Presterl T, Geiger H H, Engels C. Grain yield and kernel weight of two maize genotypes differing in nitrogen use efficiency at various levels of nitrogen and carbohydrate availability during flowering and grain filling. Plant Soil, 2005, 272: 111-123 